\title{
A transfer matrix of free vibration for exponentially functionally graded
}

\section{beams}

\author{
Zhang Kesheng ${ }^{1,2}$ a, Sun Jianpeng ${ }^{2, b}$ and Zhang Qiang ${ }^{3, c}$ \\ ${ }^{1}$ CCCC Fourth Highway Engineering Co., LTD. Beijing 100220, China \\ ${ }^{2}$ College of Civil Engineering, Xi'an University of Architecture and Technology , Xi'an 710055, China \\ a21497790@qq.com, b494779468@qq.com, cnndsjyzq@163.com
}

Keywords: Functionally graded beams, Exponential gradient, Natural frequency, Free vibration Abstract. For analyzing the free vibration of exponentially functionally graded beams a transfer matrix has been developed. The transfer matrixes of exponentially functionally graded beams which need the loop iteration are derived in closed form. The proposed transfer matrix based on the Taylor series expansion. In this study, the precise integration operations are applied and the governing differential equations and the boundary conditions of the system are transformed into a set of algebraic equations in terms of the differential transforms of the original functions. The free vibration of the exponentially functionally graded beams with general boundary conditions is computed with the proposed transfer matrix. Various numerical examples are investigated.

\section{Introduction}

Since the transfer matrix method (TMM) initially applied to solve the problems of torsion vibrations of rods by $\mathrm{H}$. Holzer[1], which a analytic transformation technique has been rapidly developed and used widely in engineering mechanics by a number of researchers. The transfer matrix method (TMM) in vibration analysis of beams was first used by Myklestad[2]. Since then, more and more researchers applied TMM to deal with vibration analysis of beam [3-5]. Wu and Chang[6], Wang [7] and Zhang [8] used TMM to analysis the free vibration of Timoshenko beam. and so fourth.

Functionally graded beams have attracted increasing attention of researchers in recent years. The material properties of functionally graded beams vary along the thickness direction or/and the length direction. For functionally graded beams with thickness-wise gradient variation, great progress has been made.

\section{A transfer matrix of free vibration}

Dynamic differential equations of bending vibration of functionally graded beam.

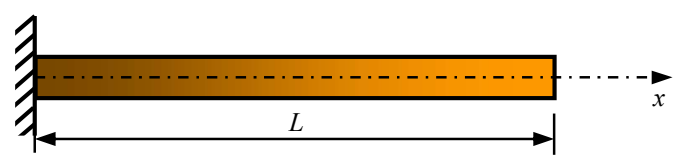

Fig.1. Schematic of an axially functionally graded beam

Let us consider the functionally graded beam in Fig.1. The length of the functionally graded beam is denoted as $\mathrm{L}$, and the axial direction is denoted as $\mathrm{x}$. The distributed mass and the bending stiffness are dependent on the axial coordinate x. For simplicity, we assume: 


$$
E I(x)=D e^{2 \delta x / L}, \rho A(x)=m e^{2 \delta x / L} .
$$

Where $\delta$ is a dimensionless gradient parameter, $\mathrm{E}$ is the Young's modulus, $\mathrm{I}(\mathrm{x})$ is the varying second-order moment of cross-sectional area, and $\mathrm{D}$ is the origin value of $\mathrm{EI}(\mathrm{x})$ at $\mathrm{x}=0, \rho$ is the mass density, $\mathrm{A}(\mathrm{x})$ is the cross-sectional area and $\mathrm{m}$ is the origin value of $\rho A(x)$ at $\mathrm{x}=0$. Notice that as $\mathrm{x}$ is raised, $\delta>0$ indicates that $\mathrm{I}(\mathrm{x})$ and $\mathrm{A}(\mathrm{x})$ increase, and $\delta<0$ indicates $\mathrm{I}(\mathrm{x})$ and $\mathrm{A}(\mathrm{x})$ decrease, respectively.

The differential equation that governs the transverse vibration of the functionally graded beams reads

$$
\frac{d^{2}}{d x^{2}}\left[D e^{2 \delta x / L} \frac{d^{2} W}{d x^{2}}\right]+m e^{2 \delta x / L} \frac{d^{2} W}{d t^{2}}=0,0<x<L
$$

The $\mathrm{W}(\mathrm{x})$ can be taken as the follow form

$$
W(x, t)=w(x) \sin \omega t .
$$

Where, $\mathrm{W}(\mathrm{x})$ is the amplitude of vibration, and $\omega$ is the circular frequency of vibration. After plugging the above expression into Eq. (2), we can get

$$
\frac{d^{2}}{d x^{2}}\left[D e^{2 \delta x / L} \frac{d^{2} w}{d x^{2}}\right]-m \omega^{2} e^{2 \delta x / L} w=0
$$

Eq. (4) can be rewritten as

$$
D\left[\left(\frac{2 \delta}{L}\right)^{2} e^{2 \delta x L L} \frac{d^{2} w}{d x^{2}}+\frac{4 \delta}{L} e^{2 \delta x L L} \frac{d^{3} w}{d x^{3}}+e^{2 \delta x L L} \frac{d^{4} w}{d x^{4}}\right]-m \omega^{2} e^{2 \delta x L L} w=0 .
$$

With $\mathrm{w}(\mathrm{x})$ at hand, the bending moment $\mathrm{M}(\mathrm{x})$, shear force $\mathrm{Q}(\mathrm{x})$ and bending angle $\psi(x)$ at any cross section are given respectively in terms of the introduced dimensionless variables by

$$
M(x)=-D e^{2 \delta x / L} \frac{d^{2} w(x)}{d x^{2}},
$$

$$
Q(x)=\frac{d M(x)}{d x},
$$

$$
\psi(x)=\frac{d w(x)}{d x} .
$$

(8)

Plug Eq. (6) and Eq. (7) into Eq. (5), we can get

$$
\left(\frac{2 \delta}{L}\right)^{2} M-\frac{4 \delta}{L} Q+D e^{2 \delta x / L} \frac{d^{4} w}{d x^{4}}-m \omega^{2} e^{2 \delta x / L} w=0,
$$

(9)

And

$$
\frac{d Q(x)}{d x}=D e^{2 \delta x / L} \frac{d^{4} w(x)}{d x^{4}} .
$$

So Eq. (6), Eq. (7) and Eq. (9) can be written as follows:

$$
\frac{d \psi(x)}{d x}=-\frac{M(x)}{D e^{2 \delta x / L}}
$$




$$
\frac{d Q(x)}{d x}=m \omega^{2} e^{2 \delta x / L} w \text {. }
$$

In vector notation Eq. (8) and Eq. (11) to Eq. (13) can be annotated as:

$$
\frac{d \chi(x)}{d x}=\eta \chi(x)
$$

Where, $\chi(x)=\{w(x), \psi(x), M(x), Q(x)\}^{\mathrm{T}}$

$$
\eta=\left[\begin{array}{cccc}
0 & 1 & 0 & 0 \\
0 & 0 & \frac{1}{D e^{2 \delta x / L}} & 0 \\
0 & 0 & 0 & 1 \\
\omega^{2} m e^{2 \delta x / L} & 0 & 0 & 0
\end{array}\right] .
$$

Precise transfer matrix method

In order to obtain a nontrivial solution of the above Eq. (14), Eq. (14) can be expressed as Biaosong:

$$
\chi_{k+1}=\Theta \chi_{k} \text {, }
$$

Where, $\Theta$ is an exponential matrix and written as follows:

$$
\Theta=\exp (\eta \Delta x) .
$$

Firstly, the distance step length is defined as

$$
s=\Delta x / 2^{N},
$$

Where, $\Delta x=x_{k+1}-x_{k}, N$ is the algorithm parameter controlling the number of total steps. Then as for distance step length $s$, the conventional algorithm for exponential matrix can be applied. Furthermore, a very small distance step $\tau$ is defined as

$$
\tau=s / 2^{\mathrm{r}},
$$

Where, $\Upsilon=20$, then $2^{N}=1,048,576$.The application of exponential matrix to Eq. (16) gives

$$
\Theta^{*}=\exp (\eta s)=[\exp (\eta \tau)]^{2 N} .
$$

As for the extremely small distance step s, the matrix exponential is evaluated by Taylor expansions

$$
\Theta^{*}(\tau)=\exp (\eta \tau) \approx \boldsymbol{I}+\eta \tau+(\eta \tau)^{2} / 2 !+\mathrm{L}+(\eta \tau)^{p} / p !=\boldsymbol{I}+\Theta_{a}^{*} .
$$

$$
\Theta_{a}^{*}=\exp (\eta \tau) \approx \boldsymbol{I}+\boldsymbol{\eta} \tau+(\eta \tau)^{2} / 2 !+\mathrm{L}+(\eta \tau)^{p} / p !
$$

Because of $\tau, \Theta_{a}^{*}$ is extremely small compared to identity matrix $\boldsymbol{I}$. In order to avoid losing 
significant digits in addition operations on computer, by noting that

$$
\left(\boldsymbol{I}+\Theta_{a}^{*}\right)^{2}=\boldsymbol{I}+2 \Theta_{a}^{*}+\Theta_{a}^{*} \times \Theta_{a}^{*} .
$$

We have

for (iter $=1$; iter $<\mathrm{N}+1$; iter ++ ) $\Theta_{a}^{*}=2 \Theta_{a}^{*}+\Theta_{a}^{*} \times \Theta_{a}^{*}$.

After the loop, the matrix exponential for a distance step length $\mathrm{s}$ is

$\Theta^{*}=\boldsymbol{I}+\Theta_{a}^{*}$.

Then the matrix exponential for the entire distance interval ${ }^{\left[x_{k}, x_{k+1}\right]}$ can be further computed by

$\Theta^{*}=\exp (\eta \Delta x)=[\exp (\eta s)]^{2 N}$

The procedures are listed as

$\bar{\Theta}^{*}=\Theta^{*}$.

(26)

for $\quad$ (iter=1; $\quad$ iter $\quad<M+1 ; \quad$ iter $\quad++) \quad \bar{\Theta}^{*}=\bar{\Theta}^{*} \times \bar{\Theta}^{*}$.

As for a series of segment problem, the sequential algorithm is to combine with the first segments and then use the new results to combine with the third segment and so forth until all segments are combined. After obtaining the results for entire interval, we can obtain the full solution at the initial point and then the original problem can be transformed into an initial value problem. With the results of $\bar{\Theta}^{*}$ for each segment, the distance marching scheme can be carried out.

\section{Boundary conditions}

In this section, we will consider several typical end supports of a beam.

The associated boundary conditions of pinned-pinned beams or simply supported beams read $\boldsymbol{w}(0)=0, \boldsymbol{M}(0)=0$,

$\boldsymbol{w}(L)=0, \boldsymbol{M}(L)=0$,

The associated boundary conditions of clamped-clamped beams read

$\boldsymbol{w}(0)=0, \psi(0)=0$,

$\boldsymbol{w}(L)=0, \psi(L)=0$,

The associated boundary conditions of clamped-free beams read

$\boldsymbol{w}(0)=0, \psi(0)=0$,

$\boldsymbol{M}(L)=0, \boldsymbol{Q}(L)=0$,

(30)

For other end supports, the associated boundary conditions were not reported here for which can be similarly read.

\section{Numerical examples}

In this section, we will consider several typical end supports of a beam. Furthermore, the natural frequencies are evaluated and mode shapes are given. The first example is a clamped-free supported functionally graded beam. The second example is a functionally graded beam with the associated 
boundary conditions of clamped-clamped. And the third example is a functionally graded beam with the associated boundary conditions of simple supported.

Fig. 2 shows a clamped-free supported functionally graded beam with square cross-section and the gradient parameter $\alpha$. The basic member properties are length $l=30 \mathrm{~m}$, cross-section $A_{0}=0.01 \mathrm{~m}^{2}$, Young's modulus $E=2.058 \times 10^{11} \mathrm{~N} \cdot \mathrm{m}^{-2}$, moments of inertia with respect to y-axis $I_{0}=8.3 \times 10^{-6}$, and density $\rho=7.860 \times 10^{3} \mathrm{~kg} \cdot \mathrm{m}^{-3}$.

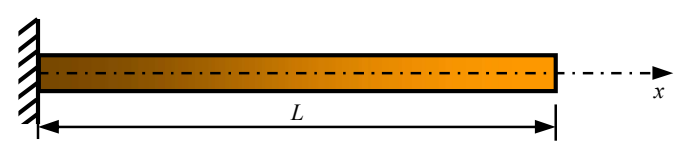

Fig.2. Functionally graded beam with clamped-free supported

In Fig. 3, the first three mode shapes of functionally graded beam with clamped-free supported are plotted which show the effects of the gradient parameter $\alpha$ on the mode shapes. However, we do not normalize the mode shapes.

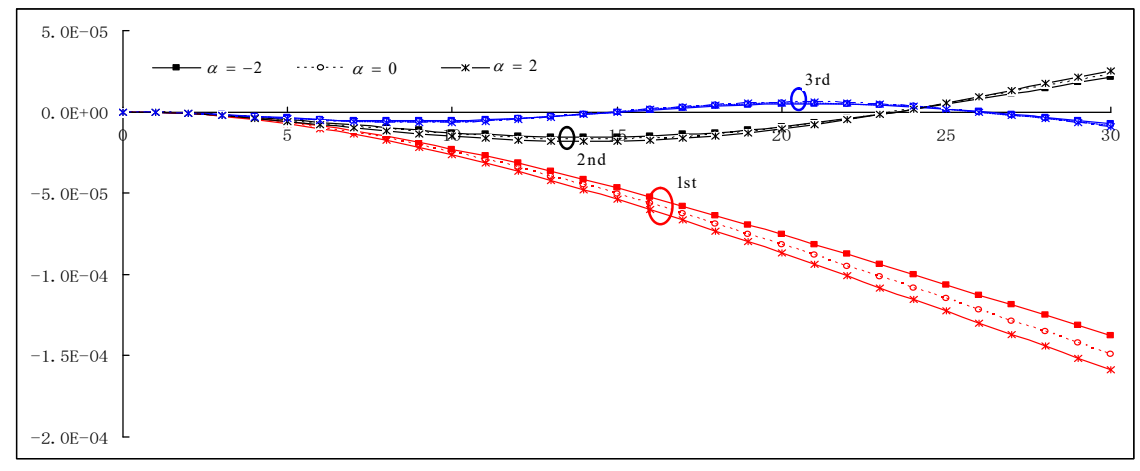

Fig.3. The first three mode shapes for exponentially graded beams with clamped-free supported with $\alpha=-2,0,2$.

It can be observed from Fig. 3 that the material gradient has a strong influence on the first mode shape and the effect becomes smaller for the higher-order vibration modes. It implies that the non-propagating components of bending waves corresponding play a significant role in determining the amplitude of the lower-order vibration mode, while their effects are gradually weak with the mode number increasing.

\section{Conclusion}

In this paper, the transfer matrix for free vibration of exponentially functionally graded beams is developed, and the free vibration of axially functionally graded beams was studied. All the results show that the material gradient has a strong influence on the first mode shape and the effect becomes smaller for the higher-order vibration modes. It implies that the non-propagating components of bending waves corresponding play a significant role in determining the amplitude of the lower-order vibration mode, while their effects are gradually weak with the mode number increasing.

\section{Acknowledgment}

The financial support of Specialized Research Fund for the Doctoral Program of Higher Education(The New Teachers)( 20136120120022) and National Natural Science Foundation of China (Grant Nos. 51408453) are much appreciated. 


\section{References}

[1] H. Holzer. Die Berechnung der Drehsenwingungen, Springer, Berlin, Germany, 1921.

[2] N. O. Myklestad. New method of calculating natural modes of coupled bending-torsion vibration of beams, Transaction of the ASME,1945;67: 61-67.

[3] W. T. Thomson. Matrix solution for the vibration of non-uniform beams, Journal of Applied Mechanics, 1950,17,337-339.

[4] D.H.Hodges,Y.Y.Chung,X.Y.Shang. Discrete transfer matrix method for non uniform rotating beams, Journal of Sound and Vibration,1994; 169(2):276-283.

[5] Khdeir AA, Reddy JN. Free vibration of cross-ply laminated beams with arbitrary boundary conditions. Int J Eng Sci, 1994 ;32(12): 71-80.

[6] Jong-Shyong Wu, Bo-Hau Chang. Free vibration of axial-loaded multi-step Timoshenko beam carrying arbitrary concentrated elements using continuous-mass transfer matrix method, European Journal of Mechanics A/Solids, 2013;38(3-4):20-37.

[7] Zhenyu Wang, Pei Zhang, and Yongqiang Zhang. Locally Resonant Band Gaps in Flexural Vibrations of a Timoshenko Beam with Periodically Attached Multioscillators, Mathematical Problems in Engineering,2013.

[8] Zhenguo Zhang, Feng Chen, Zhiyi Zhang, Hongxing Hua. Vibration analysis of non-uniform Timoshenko beams coupled with flexible attachments and multiple discontinuities, International Journal of Mechanical Sciences, 2014;80: 
